\title{
Structural and Thermophysical Properties of Cadmium Oxide
}

\author{
Purvee Bhardwaj \\ High Pressure Research Laboratory, Department of Physics, Barkatullah University, Bhopal 462026, India \\ Correspondence should be addressed to Purvee Bhardwaj, purveebhardwaj@gmail.com
}

Received 19 January 2012; Accepted 19 February 2012

Academic Editors: H. Hirao and Z. Slanina

Copyright () 2012 Purvee Bhardwaj. This is an open access article distributed under the Creative Commons Attribution License, which permits unrestricted use, distribution, and reproduction in any medium, provided the original work is properly cited.

We have studied the structural and thermophysical properties of cadmium oxide (CdO), using the Three-Body Potential (TBP) model. Phase transition pressures are associated with a sudden collapse in volume. The phase transition pressures and related volume collapses obtained from this model show a generally good agreement with available experimental others data. The thermophysical properties like molecular force constant, Debye temperature, and so forth, of CdO are also reported.

\section{Introduction}

The group of IIB-VIA oxides have presented a great deal of interest because of their applications in various technologies [1]. The semiconducting compounds of this group crystallize mostly in the zincblende (B3), wurtzite (B4), or both structures. Cadmium oxide $(\mathrm{CdO})$ is one of the binary oxides having important electronic, structural, and optical properties. Cadmium oxide occurs naturally as the rare mineral monteponite. $\mathrm{CdO}$ is a semiconductor with a band gap of $2.16 \mathrm{eV}$ at room temperature. It normally crystallizes in a cubic sodium chloride $(\mathrm{NaCl})$ rock-salt structure, with octahedral cation and anion centers. However under pressure it shows a first-order structural phase transition from $\mathrm{NaCl}$ (B1) to $\mathrm{CsCl}$ (B2) structure [2]. First-principles calculations of the crystal structures, and phase transition, and elastic properties of cadmium oxide $(\mathrm{CdO})$ have been carried out with the plane-wave pseudopotential density functional theory method by Peng et al. [3]. Liu et al. studied the B1 to $\mathrm{B} 2$ phase transition pressure at about $90.6 \mathrm{GPa}$ for $\mathrm{CdO}$ [4]. Guerrero-Moreno et al. [5] observed the ground-state properties of $\mathrm{CdO}$ with $\mathrm{B} 1$ to $\mathrm{B} 2$ structure, using the first 2 principles calculations.

We have applied the Three-Body Potential (TBP) model to the present compound to study the high pressure phase transition and other properties. The need of inclusion of three-body interaction forces was emphasized by many workers for the betterment of results [6-8]. Earlier calculations for B1-B2 transitions were based on two-body potential mainly. They concluded that possible reasons for disagreements include the failure of the two-body potential model. Since these studies were based on two-body potentials and could not explain Cauchy violations $\left(\mathrm{C}_{12} \neq \mathrm{C}_{44}\right)$. They remarked that results could be improved by including the effect of nonrigidity of ions in the model. This ThreeBody Potential (TBP) model consists of long-range Coulomb energy, three body interactions corresponding to the nearest neighbour separation, vdW (van der Waal) interaction, and energy due to the overlap repulsion represented by Hafemiester and Flygare (HF) [9] type potential and extended up to the second-neighbour ions. The purpose of this work is to investigate the structural and thermophysical properties of CdO.

\section{Potential Model and Method}

Application of pressure directly results in compression leading to the increased charge transfer (or three body interaction effect [10]) due to the deformation of the overlapping electron shell of the adjacent ions (or nonrigidity of ions) in solids.

These effects have been incorporated in the Gibbs free energy $(G=U+P V-T S)$ as a function of pressure and three-body interactions (TBI) [10], which are the most dominant among the many body interactions. Here, $U$ is the internal energy of the system equivalent to the lattice energy at temperature near zero and $S$ is the entropy. At temperature 
$T=0 \mathrm{~K}$ and pressure $(P)$ the Gibbs free energies for rock salt $(\mathrm{B} 1$, real $)$ and $\mathrm{CsCl}(\mathrm{B} 2$, hypothetical) structures are given by

$$
\begin{gathered}
G_{\mathrm{B} 1}(r)=U_{\mathrm{B} 1}(r)+P V_{\mathrm{B} 1}(r), \\
G_{\mathrm{B} 2}\left(r^{\prime}\right)=U_{\mathrm{B} 2}\left(r^{\prime}\right)+P V_{\mathrm{B} 2}\left(r^{\prime}\right) .
\end{gathered}
$$

With $V_{B 1}\left(=2.00 r^{3}\right)$ and $V_{B 2}\left(=1.54 r^{\prime 3}\right)$ as unit cell volumes for $B 1$ and $B 2$ phases, respectively. The first terms in (1) and (2) are lattice energies for $B_{1}$ and $B_{2}$ structures and they are expressed as

$$
\begin{aligned}
U_{B 1}(r)=\frac{-\alpha_{m} z^{2} e^{2}}{r}-\frac{\left(12 \alpha_{m} z e^{2} f(r)\right)}{r}-\left[\frac{C}{r^{6}}+\frac{D}{r^{8}}\right] \\
+6 b \beta_{i j} \exp \left[\frac{\left(r_{i}+r_{j}-r\right)}{\rho}\right] \\
+6 b \beta_{i i} \exp \left[\frac{\left(2 r_{i}-1.414 r\right)}{\rho}\right] \\
+6 b \beta_{j j} \exp \left[\frac{\left(2 r_{j}-1.414 r\right)}{\rho}\right], \\
U_{B 2}\left(r^{\prime}\right)=\frac{-\alpha_{m}^{\prime} z^{2} e^{2}}{r^{\prime}}-\frac{\left(16 \alpha_{m}^{\prime} z e^{2} f\left(r^{\prime}\right)\right)}{r^{\prime}}-\left[\frac{C^{\prime}}{r^{\prime 6}}+\frac{D^{\prime}}{r^{\prime 8}}\right] \\
+8 b \beta_{i j} \exp \left[\frac{\left(r_{i}+r_{j}-r^{\prime}\right)}{\rho}\right] \\
+3 b \beta_{i i} \exp \left[\frac{\left(2 r_{i}-1.154 r^{\prime}\right)}{\rho}\right] \\
+3 b \beta_{j j} \exp \left[\frac{\left(2 r_{j}-1.154 r^{\prime}\right)}{\rho}\right]
\end{aligned}
$$

with $\alpha_{m}$ and $\alpha_{m}^{\prime}$ as the Madelung constants for $\mathrm{NaCl}$ and $\mathrm{CsCl}$ structure, respectively. $\mathrm{C}\left(\mathrm{C}^{\prime}\right)$ and $\mathrm{D}\left(\mathrm{D}^{\prime}\right)$ are the overall vander der Waal coefficients of B1 (B2) phases, $\beta_{i j}(i, j=1,2)$ are the Pauling coefficients. Ze is the ionic charge and $b(\rho)$ are the hardness (range) parameters, $r\left(r^{\prime}\right)$ are the nearest neighbour separations for $\mathrm{NaCl}(\mathrm{CsCl})$ structure and $f(r)$ is the three body force parameter.

These lattice energies consists of long-range Coulomb energy (first term), three-body interactions corresponding to the nearest neighbour separation $r\left(r^{\prime}\right)$ (second term), $\mathrm{vdW}$ (van der Waal) interaction (third term), and energy due to the overlap repulsion represented by Hafemeister and Flygare (HF) type potential and extended up to the second neighbour ions (remaining terms).

\section{Results and Discussion}

The Gibb's free energies contain three model parameters

\begin{tabular}{|c|c|c|c|c|}
\hline \multirow{2}{*}{ Solid } & \multicolumn{3}{|c|}{ Phase transition pressure $(\mathrm{GPa})$} & \multirow{2}{*}{$\begin{array}{c}\text { Volume } \\
\text { collapse \% } \\
\text { Present }\end{array}$} \\
\hline & Present & Expt. & Others & \\
\hline $\mathrm{CdO}$ & 90 & $91-102^{\mathrm{a}}$ & $\begin{array}{c}102.5^{\mathrm{b}} \\
83.1^{\mathrm{b}}\end{array}$ & 6.15 \\
\hline
\end{tabular}
$[b, \rho, f(r)]$. The values of these parameters have been evaluated using the first- and second-order space derivatives of the cohesive energy $(U)$ expressed as

$$
\begin{gathered}
{\left[\frac{d U}{d r}\right]_{r=r_{0}}=0,} \\
{\left[\frac{d^{2} U}{d r^{2}}\right]_{r=r_{0}}=9 k r_{0} B,}
\end{gathered}
$$

TABLE 1: Input parameters and generated model parameters for CdO.

\begin{tabular}{lccccc}
\hline \multirow{2}{*}{ Solid } & \multicolumn{2}{c}{ Input parameters } & \multicolumn{3}{c}{ Model parameters } \\
& $r_{0}(\AA)$ & $B(\mathrm{GPa})$ & $b\left(10^{-12} \mathrm{ergs}\right)$ & $\rho(\AA)$ & $f(r)$ \\
\hline $\mathrm{CdO}$ & $2.389^{\mathrm{a}}$ & $148^{\mathrm{a}}$ & 12.5687 & 0.287 & 0.01342 \\
\hline${ }^{\mathrm{r}} \operatorname{ref}[4]$. & & & & &
\end{tabular}

TABLE 2: Phase transition and volume change of CdO.

aref [4], ${ }^{b}$ ref [5].

and following method adopted earlier [11]. Using these model parameters and the minimization technique, phase transition pressures of $\mathrm{CdO}$ have been computed. The input data of the crystal and calculated model parameters are listed in Table 1. We have followed the technique of minimization of Gibbs free energies of real and hypothetical phases. We have minimized $G_{B 1}(r)$ and $G_{B 2}\left(r^{\prime}\right)$ given by (3) and (4) at different pressures in order to obtain the interionic separations $r$ and $r^{\prime}$ corresponding to $B_{1}$ and $B_{2}$ phases associated with minimum energies. The factor $\Delta G\left[G_{B 1}(r) \sim G_{B 2}\left(r^{\prime}\right)\right]$ plays an important role in stability of structures. The phase transition occurs when $\Delta G$ approaches zero $(\Delta G \longrightarrow 0)$. The phase transition pressure $\left(P_{t}\right)$ is the pressure at which $\Delta G$ approaches zero. At $P_{t}$ these compounds undergo a (B1-B2) transition associated with a sudden collapse in volume showing a first-order phase transition. Figure 1 shows our present computed phase transition pressure for $\mathrm{NaCl}$-type $(B 1)$ to CsCl-type (B2) structures in $\mathrm{CdO}$ at $90 \mathrm{GPa}$. The present phase transition pressure is illustrated by arrow in Figure 1. The calculated values of phase transition pressure have been listed in Table 2 and compared with their experimental and other theoretical results. It is interesting to note from Table 2 and Figure 1 that the phase transition pressures $\left(P_{t}\right)$, obtained from our model, are in general in closer agreement with experimental data [4] and match equally well with other theoretical results [5].

The compression curves are plotted in Figure 2. The values of the volume collapses $(-\Delta V(p) / V(0))$ are depicted in Table 2. The experimental and theoretical values of volume collapses are not available for the present compounds. It is clear that during the phase transition from $\mathrm{NaCl}$ to $\mathrm{CsCl}$, the volume discontinuity in pressure volume phase diagram identifies the occurrence of first-order phase transition and the same trend as the other theoretical approach. In Figure 2 the pressure versus volume graph has been plotted.

In additional to know the behaviour of the interionic distance with pressure for the present oxide, we present the variation of nearest-neighbor (nn) and next-nearest neighbor (nnn) distances for both the $B 1$ and $B 2$ phases with pressure in Figure 3. The interionic distances of the present oxide decrease on increasing the pressure. The open 


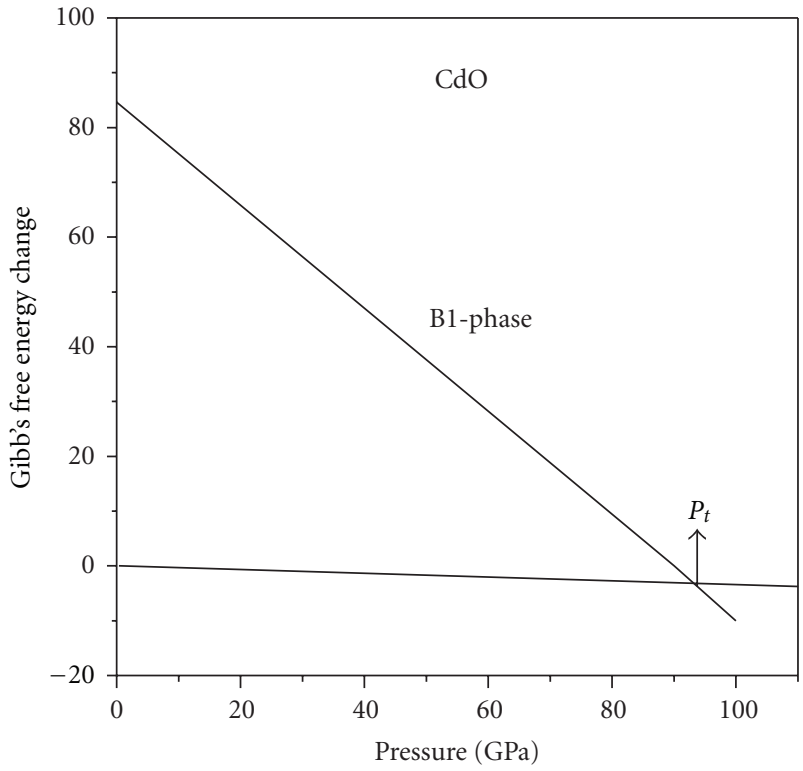

Figure 1: Variation of Gibb's free energy with pressure for CdO.

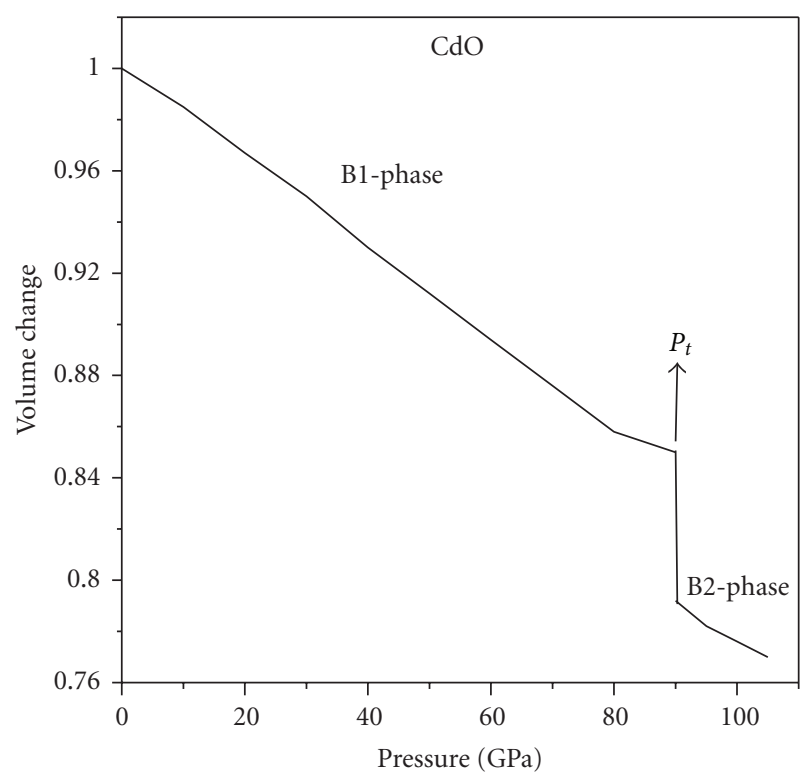

Figure 2: Variation of volume change $V_{P} / V_{0}$ with pressure for CdO.

circles represent the nearest-neighbor (nn) and solid circles represent next nearest neighbor (nnn) distance in Figure 3 for $\mathrm{CdO}$.

To further increase the applicability of our model, we have calculated the molecular force constant $(f)$, infrared absorption frequency $\left(v_{0}\right)$, Debye temperature $\left(\theta_{D}\right)$, and Grunneisen parameter $(\gamma)$ which are directly derived from the cohesive energy, $U(r)$.

The compressibility is well known to be given by

$$
\beta=\frac{3 K r_{0}}{f}
$$

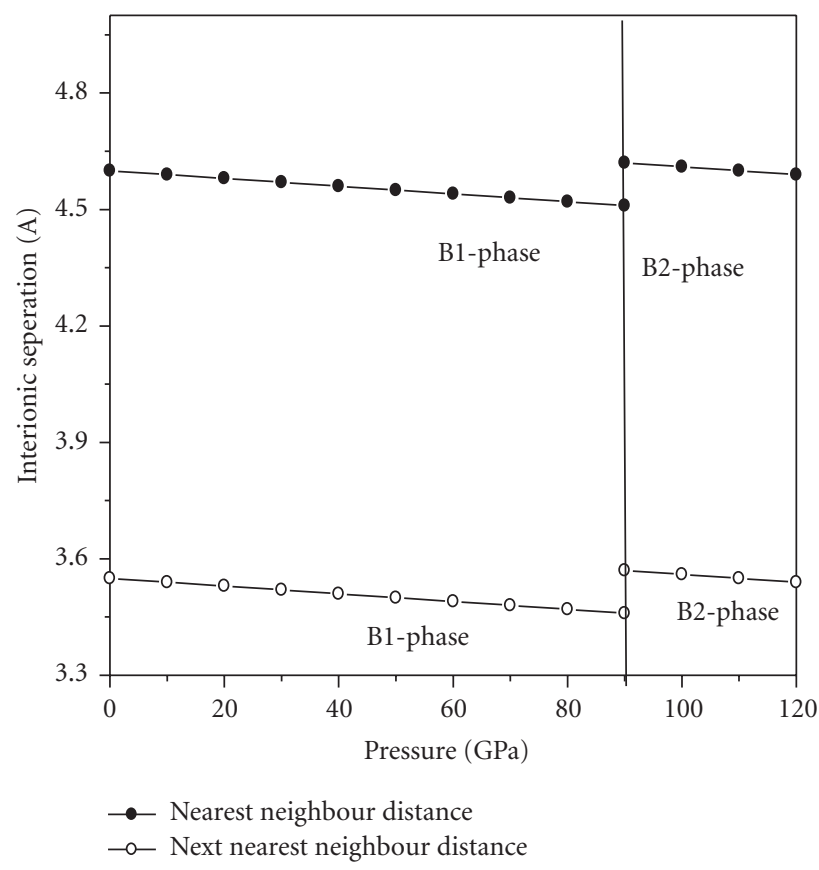

FIgURE 3: Variation of interionic distance with pressure for CdO.

TABle 3: Thermophysical properties of CdO.

\begin{tabular}{lcccc}
\hline Solid & $f\left(10^{4} \mathrm{dyn} / \mathrm{cm}\right)$ & $v_{0}\left(10^{12} \mathrm{~Hz}\right)$ & $\theta_{D}(\mathrm{~K})$ & $\gamma$ \\
\hline CdO & & & & \\
Present & 10.1571 & 7.0968 & 328 & 1.0421 \\
Others & - & - & $336.5^{\mathrm{a}}$ & - \\
\hline${ }^{\mathrm{a}}$ ref [3]. & & & &
\end{tabular}

in terms of molecular force constants

$$
f=\frac{1}{3}\left[U_{k k^{\prime}}^{S R}(r)+\frac{2}{r} U_{k k^{\prime}}^{S R}(r)\right]_{r=r_{0}} .
$$

With $U_{k k^{\prime}}^{S R}(r)$ as the short range nearest neighbour $\left(k \neq k^{\prime}\right)$ part of $U(r)$ given by the last three terms in (3) and (4). This force constant $f$ leads to the infrared absorption frequency with the knowledge of the reduced mass $(\mu)$ of the crystals. The thermal expansion coefficient $\left(\alpha_{v}\right)$ can be calculated with the knowledge of specific heat $\left(C_{v}\right)$. The expressions have been given in our earlier paper [12].

We have calculated the thermophysical properties of CdO. The thermophysical properties provide us the interesting information about the substance. The Debye characteristic temperature $\theta_{D}$ reflects its structure stability, the strength of bonds between its separate elements, structure defects availability, and its density. The calculated thermophysical properties have been listed in Table 3. Due to the lack of experimental and theoretical data, we could not compare them. We have compared the value of Debye temperature with theoretical results provided by Peng et al. [3]. Our result shows the same trend as reported by others. As to the best of our knowledge the value of the thermal properties for present compounds have not yet been measured or calculated, hence our results can serve as a prediction for future investigations. 
In view of the overall attainments, it may be concluded that there is generally a good agreement of Three-Body Potential (TBP) model with the available experimental and theoretical values. Finally, it may be concluded that the present model has successfully predicted the compression curves and phase diagrams giving the phase transition pressures, associated volume collapses, and elastic properties correctly for cadmium oxide.

\section{References}

[1] A. Schleife, F. Fuchs, J. Furthmüller, and F. Bechstedt, "Firstprinciples study of ground- and excited-state properties of $\mathrm{MgO}, \mathrm{ZnO}$, and CdO polymorphs," Physical Review B, vol. 73, no. 24, Article ID 245212, 14 pages, 2006.

[2] H. Liu, J. S. Tse, and H. Mao, "Stability of rocksalt phase of zinc oxide under strong compression: synchrotron $\mathrm{x}$ ray diffraction experiments and first-principles calculation studies," Journal of Applied Physics, vol. 100, no. 9, Article ID 093509, 5 pages, 2006.

[3] F. Peng, Q. Liu, H. Fu, and X. Yang, "First-principles calculations on phase transition and elasticity of CdO under pressure," Solid State Communications, vol. 148, no. 1-2, pp. 6-9, 2008.

[4] H. Liu, H. Mao, M. Somayazulu, Y. Ding, Y. Meng, and D. Häusermann, "B1-to-B2 phase transition of transition-metal monoxide CdO under strong compression," Physical Review B, vol. 70, no. 9, Article ID 094114, 5 pages, 2004.

[5] R. J. Guerrero-Moreno and N. Takeuchi, "First principles calculations of the ground-state properties and structural phase transformation in CdO," Physical Review B, vol. 66, no. 20, Article ID 205205, 6 pages, 2002.

[6] C. E. Sims, G. D. Barrera, and N. L. Allan, "Thermodynamics and mechanism of the B1-B2 phase transition in group-I halides and group-II oxides," Physical Review B, vol. 57, no. 18, pp. 11164-11172, 1998.

[7] B. S. Rao and S. P. Sanyal, "High pressure structural phase transition in BaSe and BaTe," Physica Status Solidi B, vol. 165, no. 2, pp. 369-375, 1991.

[8] S. Froyen and M. L. Cohen, "Structural properties of $\mathrm{NaCl}$ and $\mathrm{KCl}$ under pressure," Journal of Physics C, vol. 19, no. 15, pp. 2623-2632, 1986.

[9] D. W. Hafemiester and W. H. Flygare, "Outer-shell overlap integrals as a function of distance for halogen-halogen halogen-alkali and alkali-alkali ions in the alkali halide lattice," Journal of Chemical Physics, vol. 43, no. 3, pp. 795-800, 1965.

[10] R. K. Singh, "Many body interactions in binary ionic solids," Physics Reports, vol. 85, no. 5, pp. 259-401, 1982.

[11] P. Bhardwaj, S. Singh, and N. K. Gaur, "Structural and elastic properties of barium chalcogenides $(\mathrm{BaX}, \mathrm{X}=\mathrm{O}, \mathrm{Se}, \mathrm{Te})$ under high pressure," Central European Journal of Physics, vol. 6, no. 2, pp. 223-229, 2008.

[12] P. Bhardwaj, S. Singh, and N. K. Gaur, "Structural, elastic and thermophysical properties of divalent metal oxides with $\mathrm{NaCl}$ structure," Materials Research Bulletin, vol. 44, no. 6, pp. 13661374, 2009. 

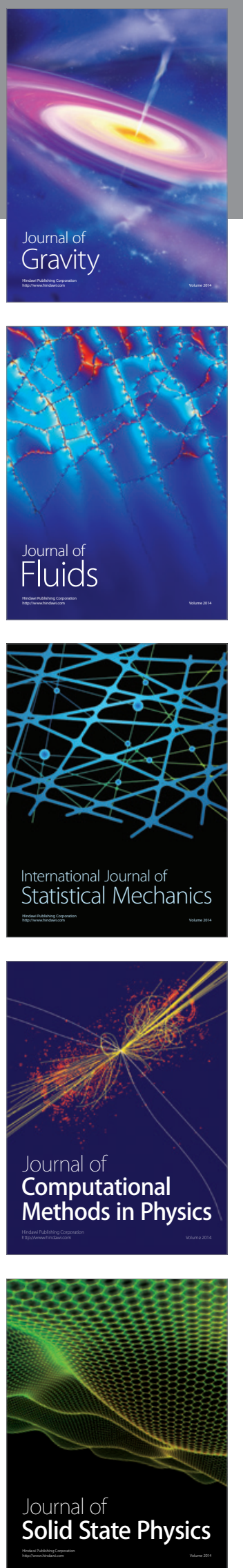
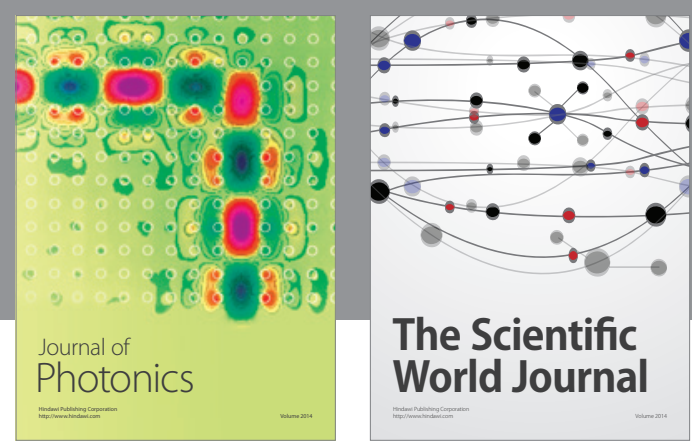

The Scientific World Journal

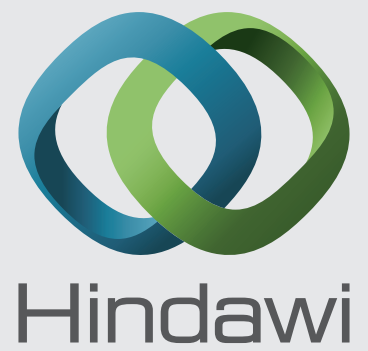

Submit your manuscripts at http://www.hindawi.com
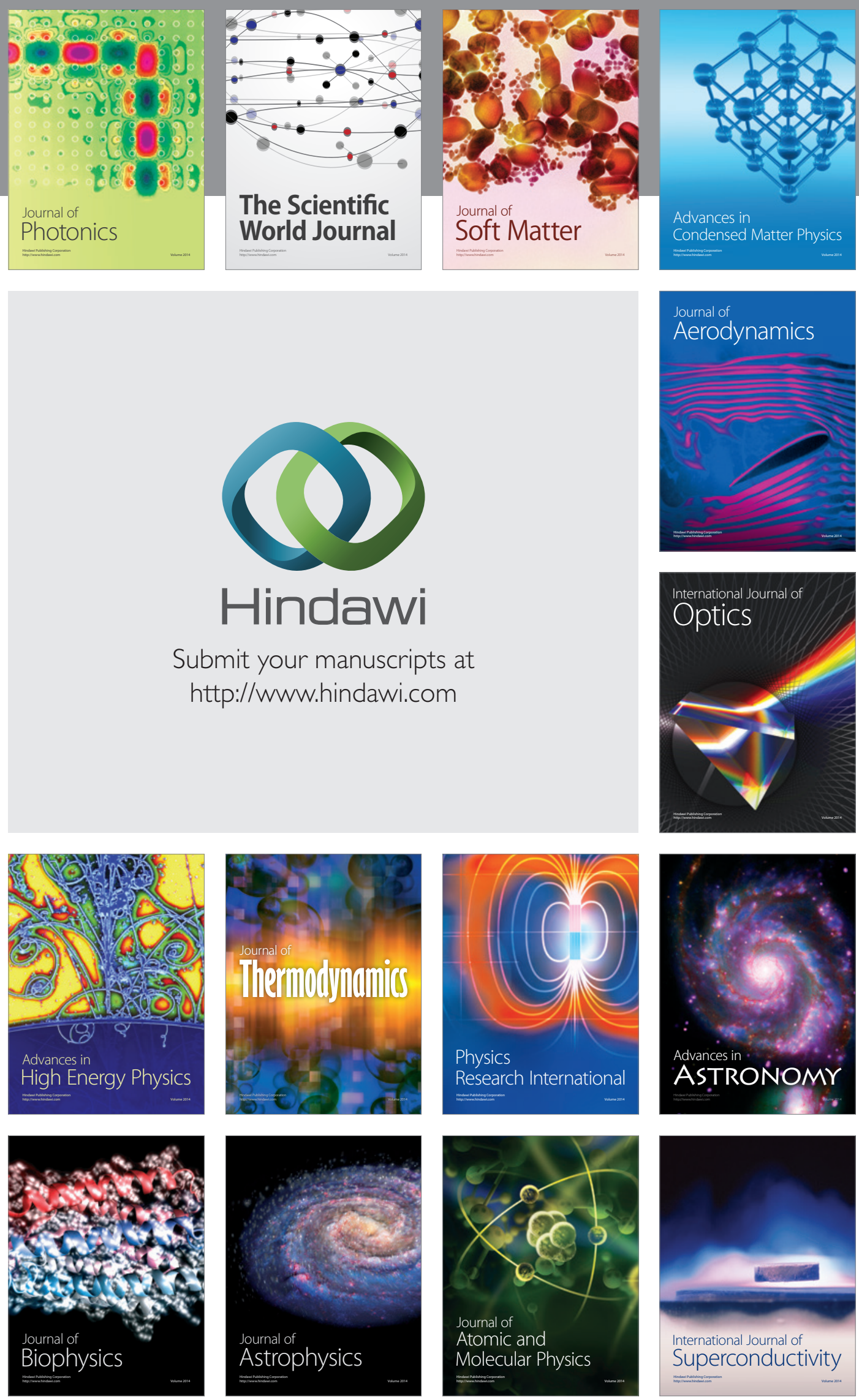
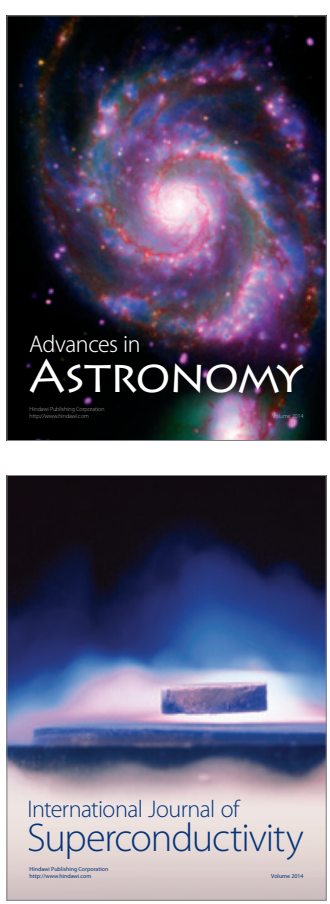